\title{
On the Keller-Osserman conjecture in one dimensional case
}

\author{
Lin-Lin Wang and Yong-Hong Fan*
}

"Correspondence: fanyh_1993@sina.com

School of Mathematics and Statistics Science, Ludong University, Yantai, Shandong 264025, P.R. China

\begin{abstract}
A sufficient and necessary condition for existence of solution for the boundary blow-up problem in one dimensional case is obtained. This problem can be seen as the Keller-Osserman conjecture, which comes from the study on elliptic equations.
\end{abstract}

Keywords: Keller-Osserman conjecture; boundary blow-up problem; symmetrical method

\section{Introduction}

The boundary blow-up problem

$$
\left\{\begin{array}{l}
\Delta u(x)=f(u(x)), \quad x \in \Omega, \\
\left.u\right|_{\partial \Omega}=+\infty
\end{array}\right.
$$

where $\Omega$ is a bounded domain in $R^{N}(N \geq 2)$, arises in many fields, such as the theory of automorphic functions and Riemann surfaces of constant negative curvature, the study of the electric potential in a glowing hollow metal body, etc. In 1916, this type of problem was first studied by Bieberbach (see [1]). A very famous result is the following.

Theorem A Assume that $f(0)=0, f^{\prime}(t)$ is continuous and $f^{\prime}(t) \geq 0$ for all $t \geq 0$, then (1.1) has a solution if and only if

$$
\int_{1}^{+\infty}\left(\int_{0}^{t} f(s) d s\right)^{-1 / 2} d t<+\infty .
$$

The condition (1.2) plays an important role in the study of the boundary blow-up problem, it was first proposed by Keller [2] and Osserman [3], thus lately, this condition was called the Keller-Osserman condition. Also see [4] and [5]. For later investigations on boundary blow-up problems we refer to [6-10] etc. An interesting problem is the following.

Keller-Osserman conjecture When $N=1$, the conclusion of Theorem $\mathrm{A}$ is also true.

For the Keller-Osserman conjecture, see Anuradha et al. (see [11]) who first considered the following autonomous two point boundary value problem: 


$$
\left\{\begin{array}{l}
-u^{\prime \prime}(t)=\lambda f(u(t)) ; \quad 0<t<1, \\
\lim _{t \rightarrow 0^{+}} u(t)=\lim _{t \rightarrow 1^{-}} u(t)=+\infty .
\end{array}\right.
$$

Using the quadratic method, they gave some necessary, sufficient conditions for the existence of nonnegative solutions. As they said, 'The gap between the class of functions that satisfy the necessary condition but not the sufficient condition is quite small.' In their paper, they gave some examples to illustrate their conclusions.

Remark 1 Anuradha et al. [11], Remarks 3.1 and 3.2, showed that, to problem (1.3):

(i) for $f(u)=-u(\ln u+1)$, there are no solutions,

(ii) for $f(u)=-u\left[2(\ln u)^{3}+3(\ln u)^{2}\right]$, there exist solutions for some $\lambda>0$.

It is easy to verify that $f(u)=-u(\ln u+1)$ does not satisfy the Keller-Osserman condition (1.2), but $f(u)=-u\left[2(\ln u)^{3}+3(\ln u)^{2}\right]$ satisfies this Keller-Osserman condition.

Recently, Wang [12] used the same method as Anuradha's and generalized the results of Anuradha's; he obtained a more suitable condition for the existence of solutions for (1.3). The gap between the class of functions that satisfy the necessary condition but not the sufficient condition becomes smaller than that of Anuradha's, but we still have a distance to Keller-Osserman's conjecture. Later Zhang [13] considered this problem again. In this paper, we want to study problem (1.3) again and solve this conjecture.

In this paper, we investigate the following boundary blow-up problem:

$$
\left\{\begin{array}{l}
u^{\prime \prime}(t)=f(u(t)) ; \quad 0<t<1, \\
\lim _{t \rightarrow 0^{+}} u(t)=\lim _{t \rightarrow 1^{-}} u(t)=+\infty,
\end{array}\right.
$$

where $f(t) \geq 0, f(t)$ is continuous and monotone increasing for all $t \geq 0$. For convenience, we set $u(1-0)=+\infty$ if $\lim _{t \rightarrow 1^{-}} u(t)=+\infty$, for short, denote $u(1)$.

First we introduce the definition of regularly varying which can be found in [14].

Definition 1 A positive measurable function $R$ defined on $[D,+\infty)$, for some $D>0$, is called regularly varying (at infinity) with index $q \in \mathbb{R}$, written $R \in \mathbb{R}_{q}$, if for all $\xi>0$,

$$
\lim _{u \rightarrow \infty} R(\xi u) / R(u)=\xi^{q} .
$$

When the index of regular variation $q$ is zero, we say that the function is slowly varying.

\section{Remark}

(a) Any function $R \in \mathbb{R}_{q}$ can be written in terms of a slowly varying function. Indeed, set $R(u)=u^{q} L(u)$, then $L$ varies slowly.

(b) For any $m>0, u^{m} L(u) \rightarrow+\infty, u^{-m} L(u) \rightarrow 0$ as $u \rightarrow+\infty$.

Here come our main results.

Theorem 1 If $f(0)=0$, then equation (1.4) has a positive solution if and only if the KellerOsserman condition (1.2) holds true.

Theorem 2 Iff $(0)>0$, then equation (1.4) has a positive solution if and only if the KellerOsserman condition (1.2) and $\max _{a \geq 0} g(a) \geq \frac{\sqrt{2}}{2}$ hold true, where the function $g$ is defined in (2.3). 
Theorem 3 Assume that $f(0)=0$ and $f(t) \in \mathbb{R}_{q}, q>1$. Then the solution $u(t)$ of $(1.4)$ satisfies

$$
\lim _{t \rightarrow \partial I} \frac{u(t)}{\phi(\delta(t))}=1
$$

where

$$
\begin{aligned}
& \delta(t)=\operatorname{dist}(t, \partial I), \quad I=[0,1], \quad \phi(s)=\psi^{-1}(s), \quad F(t)=\int_{0}^{t} f(s) d s \quad \text { and } \\
& \psi(s)=\int_{s}^{+\infty} \frac{1}{\sqrt{2 F(t)}} d t .
\end{aligned}
$$

Theorem 4 Assume that $f(0)=0, \frac{f(u)}{u}$ is increasing on $(0,+\infty)$ and $f(t) \in \mathbb{R}_{q}, q>1$. Then equation (1.4) has only one positive solution.

From Theorem 1, we can easily obtain the following.

Corollary 1 If $f(0)=0$, then for any $\lambda>0$, equation (1.3) has a positive solution if and only if the Keller-Osserman condition (1.2) holds true.

Corollary 1 generalizes Theorem 3.1, Theorem 3.2 and Theorem 3.6 in [11], also generalizing Theorem 2.1 and Theorem 2.2 in [12].

\section{The proof of main results}

In order to prove our main results, we need some lemmas, presented below.

Lemma 1 If $u(t)$ is a solution of $(1.4)$, then $u(1-t)$ is also a solution of (1.4).

The proof of this lemma is trivial, we omit it here.

Lemma 2 If $u(t)$ is a solution of (1.4), then there exists only one point $t_{0} \in(0,1)$ such that $u^{\prime}\left(t_{0}\right)=0$, in fact, $t_{0}=\frac{1}{2}$.

The proof of this lemma can be obtained by the generalized Rolle theorem. $t_{0}=\frac{1}{2}$ can be obtained easily by Lemma 1 .

Lemma 3 If $u(t) \in C^{2}\left[\frac{1}{2}, 1\right)$ is a solution of the following problem:

$$
\left\{\begin{array}{l}
u^{\prime \prime}(t)=f(u(t)) ; \quad \frac{1}{2} \leq t<1, \\
u^{\prime}\left(\frac{1}{2}\right)=0, \quad u(1)=+\infty,
\end{array}\right.
$$

then

$$
w(t)= \begin{cases}u(t), & t \in\left(0, \frac{1}{2}\right) ; \\ u(1-t), & t \in\left[\frac{1}{2}, 1\right),\end{cases}
$$

is a solution of (1.4), in the contrary case, the conclusion also holds true. 
Proof The proof is trivial, we omit it.

Lemma 4 Assume that the Keller-Osserman condition holds true. Iff $(0)>0$, then the function

$$
g(a)=\int_{a}^{+\infty}\left(\int_{a}^{t} f(s) d s\right)^{-1 / 2} d t
$$

is well defined on the interval $[0,+\infty)$ and satisfies

$$
\lim _{a \rightarrow+\infty} g(a)=0
$$

If $f(0)=0$, then the function $g(a)$ is well defined on the interval $(0,+\infty)$ and satisfies

$$
\lim _{a \rightarrow 0^{+}} g(a)=+\infty, \quad \lim _{a \rightarrow+\infty} g(a)=0 .
$$

Proof Notice that $f(s)$ is increasing, thus

$$
f(a)(t-a) \leq \int_{a}^{t} f(s) d s \leq f(t)(t-a), \quad \text { for } t \geq a .
$$

Therefore

$$
[f(t)(t-a)]^{-1 / 2} \leq\left(\int_{a}^{t} f(s) d s\right)^{-1 / 2} \leq[f(a)(t-a)]^{-1 / 2}
$$

set

$$
g(a)=I(a, b)+J(a, b)
$$

where

$$
I(a, b)=\int_{a}^{b}\left(\int_{a}^{t} f(s) d s\right)^{-1 / 2} d t, \quad J(a, b)=\int_{b}^{+\infty}\left(\int_{a}^{t} f(s) d s\right)^{-1 / 2} d t, \quad b>a .
$$

By the Keller-Osserman condition, we know that $J(a, b)<+\infty$.

If $f(0)>0$, then

$$
I(a, b) \leq \int_{a}^{b}[f(a)(t-a)]^{-1 / 2} d t \leq 2 \sqrt{\frac{b-a}{f(a)}}<+\infty
$$

which means that the function $g(a)$ is well defined on the interval $[0,+\infty)$.

If $f(0)=0$, notice that $f^{\prime}(0)$ exists, thus there exist some positive constants $c$ and $k$ such that $f(t) \leq k t, c<b$ for $t \in[0, c]$, therefore

$$
I(0, b) \geq \int_{0}^{c}[f(t) t]^{-1 / 2} d t \geq k^{-1 / 2} \int_{0}^{c} \frac{1}{t} d t=+\infty,
$$

which means that the function $g(a)$ is well defined on the interval $(0,+\infty)$ (equation $(2.4)$ holds for any $a>0$ ) and $\lim _{a \rightarrow 0^{+}} g(a)=+\infty$. 
From the Keller-Osserman condition (1.2) and noticing that $\left(\int_{0}^{t} f(s) d s\right)^{-1 / 2}$ is monotone decreasing, we have

$$
\lim _{t \rightarrow+\infty} t\left(\int_{0}^{t} f(s) d s\right)^{-1 / 2}=0
$$

Hence

$$
\lim _{t \rightarrow+\infty} t^{-2} \int_{0}^{t} f(s) d s=+\infty
$$

therefore

$$
\lim _{t \rightarrow+\infty} \frac{f(t)}{t}=+\infty
$$

by (2.4), we get

$$
\lim _{a \rightarrow+\infty} I(a, 2 a)=0
$$

Note that

$$
J(a, b)=\int_{b}^{+\infty}\left(\int_{a}^{t} f(s) d s\right)^{-1 / 2} d t<+\infty
$$

then

$$
\lim _{a \rightarrow+\infty} J(a, 2 a)=0
$$

therefore

$$
\lim _{a \rightarrow+\infty} g(a)=0
$$

Lemma 5 If $f(0)=0$, then equation (2.1) has a solution if and only if the Keller-Osserman condition holds true.

Proof Sufficiency. Conversely, suppose that there is no solution $u(t)$. Given any real number $a$, let $u(t)$ be a solution of the following problem:

$$
\left\{\begin{array}{l}
u^{\prime \prime}(t)=f(u(t)) ; \quad \frac{1}{2} \leq t<1, \\
u^{\prime}\left(\frac{1}{2}\right)=0, \quad u\left(\frac{1}{2}\right)=a .
\end{array}\right.
$$

Its maximal existence interval is $\left[\frac{1}{2}, r_{a}\right)$, where $r_{a}$ such that $\lim _{t \rightarrow r_{a}^{-}} u(t)=+\infty$ and $\frac{1}{2}<r_{a}$ and $r_{a} \neq 1$. Notice that (2.6) implies that

$$
u^{\prime}(t)=\sqrt{2 \int_{a}^{u(t)} f(s) d s} \text { for } t \in\left[\frac{1}{2}, r_{a}\right) \text {, }
$$


here we used the inequality $u^{\prime}(t) \geq 0$ for $t \in\left[\frac{1}{2}, r_{a}\right)$. From (2.7), we have

$$
\int_{a}^{+\infty}\left(\int_{a}^{t} f(s) d s\right)^{-1 / 2} d t=\sqrt{2}\left(r_{a}-\frac{1}{2}\right)
$$

By Lemma 4 and the zero theorem, we know that there exists $a_{0}>0$ such that

$$
g\left(a_{0}\right)=\frac{\sqrt{2}}{2}
$$

then (2.8) implies that $r_{a_{0}}=1$, which is a contradiction.

Necessity. Assume that

$$
\int_{1}^{+\infty}\left(\int_{0}^{t} f(s) d s\right)^{-1 / 2} d t=+\infty
$$

then for any $a>0$, we have $g(a)=+\infty$, by (2.8), we know that equation (2.1) has no solution, which is a contradiction.

From (2.8) and Lemma 4, we can easily obtain the following.

Lemma 6 If $f(0)>0$, then equation (2.1) has a solution if and only if the Keller-Osserman condition and $\max _{a \geq 0} g(a) \geq \frac{\sqrt{2}}{2}$ are all satisfied.

Proof of Theorem 1 From Lemma 3 and Lemma 5, the conclusion of Theorem 1 is obvious.

Proof of Theorem 2 From Lemma 3 and Lemma 6, we can easily get it.

\section{Asymptotical stability}

In this section, we want to study the behavior of large solutions near the boundary and the uniqueness of the solution. We start with the following comparison theorem.

Lemma 7 (Comparison theorem) Assume that $f(t) \geq 0, f(t): \mathbb{R} \rightarrow \mathbb{R}$ is continuous and monotone increasing. Let $u(t), v(t) \in C^{2}\left[\frac{1}{2}, 1\right)$ such that

$$
\begin{aligned}
& u^{\prime \prime}(t) \leq f(u(t)), \quad t \in\left[\frac{1}{2}, 1\right), \\
& v^{\prime \prime}(t) \geq f(v(t)), \quad t \in\left[\frac{1}{2}, 1\right), \\
& u^{\prime}\left(\frac{1}{2}\right) \leq v^{\prime}\left(\frac{1}{2}\right), \quad u(1) \geq v(1),
\end{aligned}
$$

then $u(t) \geq v(t)$ for all $t \in\left[\frac{1}{2}, 1\right]$.

Proof Assume that it is false, then there exists some $t_{0}<t_{1}$ in $\left[\frac{1}{2}, 1\right)$ such that $u\left(t_{0}\right)<v\left(t_{0}\right)$, $u^{\prime}\left(t_{0}\right)=v^{\prime}\left(t_{0}\right), u(t) \leq v(t)$ and $u^{\prime}(t) \geq v^{\prime}(t)$ for $t \in\left[t_{0}, t_{1}\right]$. Let $w(t)=u(t)-v(t), t \in\left[t_{0}, t_{1}\right]$, then (3.1) and (3.2) imply that

$$
w^{\prime \prime}(t) \leq f(u(t))-f(v(t))
$$


Multiplying $w^{\prime}(t)$ to both sides of (3.3), we have

$$
\left(\frac{1}{2}\left[w^{\prime}(t)\right]^{2}\right)^{\prime} \leq[f(u(t))-f(v(t))] w^{\prime}(t) \quad \text { for } t \in\left[t_{0}, t_{1}\right]
$$

integrating from $t_{0}$ to $t\left(t \in\left[t_{0}, t_{1}\right]\right)$, we get

$$
\frac{1}{2}\left[w^{\prime}(t)\right]^{2} \leq \int_{t_{0}}^{t}[f(u(t))-f(v(t))] w^{\prime}(t) d t<0,
$$

which is a contradiction.

Remark In Lemma 7, if we replace $\frac{1}{2}$ and 1 by any other real numbers $a$ and $b(a<b)$, then the conclusion is also true.

Corollary 2 (Comparison theorem) Assume that $f: \mathbb{R} \rightarrow \mathbb{R}$ is continuous and monotone increasing with $f(0)=0$. Let $u(t) \in C^{2}\left[\frac{1}{2}, 1\right)$ such that

$$
\begin{aligned}
& u^{\prime \prime}(t) \leq f(u(t)), \quad t \in\left[\frac{1}{2}, 1\right), \\
& u^{\prime}\left(\frac{1}{2}\right) \leq 0, \quad u(1) \geq 0,
\end{aligned}
$$

then $u(t) \geq 0$ for all $t \in\left[\frac{1}{2}, 1\right]$.

Proof In Lemma 7, let $v(t) \equiv 0$, then we can immediately get the conclusion.

Proof of Theorem 3 First, we claim that if $h \in \mathbb{R}_{p}$ is continuous and nonnegative on $[a,+\infty)$, here $p<-1$, then $h \in L^{1}([a,+\infty))$. To see this, suppose that $h \notin L^{1}([a,+\infty))$, then $\lim _{s \rightarrow+\infty} H(s)=\int_{a}^{+\infty} h(s) d s=\infty$, by the L'Hospital rule, we have

$$
\lim _{t \rightarrow \infty} \frac{H(\xi t)}{H(t)}=\xi^{p+1}
$$

Notice that $H$ is increasing, it follows that $\xi \rightarrow \xi^{p+1}$ is increasing as well, therefore $p \geq-1$, which contradicts $p<-1 . f(t) \in \mathbb{R}_{q}$ implies $F(t) \in \mathbb{R}_{q+1}$ (by the L'Hospital rule), hence $F^{-1 / 2}(t) \in R_{-\frac{q+1}{2}}$. Notice that $q>1$ implies $-\frac{q+1}{2}<-1$, then the above claim shows that $F^{-1 / 2}(t) \in L^{1}([a,+\infty))$, that is, the Keller-Osserman condition (1.2) holds true, then from Theorem 1, we know that the solution of (1.4) exists, by Corollary 2, these solutions (this solution) $u(t)$ satisfy (satisfies) $u(t) \geq 0$. Then (2.6) implies that

$$
u^{\prime}(t)=\sqrt{2 \int_{u\left(\frac{1}{2}\right)}^{u(t)} f(s) d s .}
$$

From (3.4), we have

$$
u^{\prime}(t)=\sqrt{2\left(\int_{0}^{u(t)} f(s) d s-\int_{0}^{u\left(\frac{1}{2}\right)} f(s) d s\right)}
$$


thus

$$
\frac{u^{\prime}(t)}{\sqrt{2 \int_{0}^{u(t)} f(s) d s}}=\sqrt{1-\frac{\int_{0}^{u\left(\frac{1}{2}\right)} f(s) d s}{\int_{0}^{u(t)} f(s) d s}},
$$

for any boundary blow-up solution $u(t)$ of (1.4), we can easily obtain

$$
\lim _{t \rightarrow 1^{-}} \frac{\int_{0}^{u\left(\frac{1}{2}\right)} f(s) d s}{\int_{0}^{u(t)} f(s) d s}=0 .
$$

Then (3.5) implies that

$$
\psi(u(t))=\int_{t}^{1}(1+o(1)) d t=(1-t)(1+o(1))
$$

therefore

$$
u(t)=\phi(\beta(t)(1-t))
$$

where $\beta(t)$ satisfies

$$
\lim _{t \rightarrow 1^{-}} \beta(t)=1 \quad \text { and } \quad \beta(t)<1 .
$$

In order to prove (1.5), we only need to prove

$$
\lim _{t \rightarrow 1^{-}} \frac{\phi(\beta(t)(1-t))}{\phi(1-t)}=1
$$

From (3.6), we see, for any $\varepsilon>0$, that there exists some $\delta<1 / 2$ such that

$$
1-\varepsilon<\beta(t)<1 \quad \text { for } 1-\delta<t<1 \text {. }
$$

Notice that

$$
\phi^{\prime}(s)=-\sqrt{2 F(\phi(s))}<0,
$$

thus

$$
\phi(1-t) \leq \phi(\beta(t)(1-t)) \leq \phi((1-t)(1-\varepsilon))
$$

which implies that

$$
1 \leq \frac{\phi(\beta(t)(1-t))}{\phi(1-t)} \leq \frac{\phi((1-t)(1-\varepsilon))}{\phi(1-t)} .
$$

In the following, we prove that $\phi(t) \in \mathbb{R}_{2 /(1-q)}$ (at zero) provided that $f(t) \in \mathbb{R}_{q}$. In fact, if $f(t) \in \mathbb{R}_{q}$, then

$$
\lim _{t \rightarrow+\infty} \frac{F(\xi t)}{F(t)}=\lim _{t \rightarrow+\infty} \frac{\xi F^{\prime}(\xi t)}{F^{\prime}(t)}=\lim _{t \rightarrow+\infty} \frac{\xi f(\xi t)}{f(t)}=\xi^{q+1},
$$


which implies that

$$
\lim _{t \rightarrow+\infty} \frac{\psi(\xi t)}{\psi(t)}=\lim _{t \rightarrow+\infty} \frac{\xi \psi^{\prime}(\xi t)}{\psi^{\prime}(t)}=\lim _{t \rightarrow+\infty} \frac{\xi \sqrt{F(t)}}{\sqrt{F(\xi t)}}=\xi^{\frac{1-q}{2}}
$$

therefore

$$
\lim _{t \rightarrow+\infty} \frac{\phi\left(\xi^{(1-q) / 2} \psi(t)\right)}{t}=\xi
$$

thus

$$
\lim _{t \rightarrow 0^{+}} \frac{\phi(\xi t)}{\phi(t)}=\lim _{y \rightarrow+\infty} \frac{\phi(\xi \psi(y))}{y}=\lim _{y \rightarrow+\infty} \frac{\phi\left(\sigma^{(1-q) / 2} \psi(y)\right)}{y}=\sigma=\xi^{\frac{2}{1-q}} .
$$

So

$$
\lim _{t \rightarrow 1^{-}} \frac{\phi((1-t)(1-\varepsilon))}{\phi(1-t)}=(1-\varepsilon)^{\frac{2}{1-q}}
$$

letting $\varepsilon \rightarrow 0^{+}$, and then by (3.8), (3.7) holds true.

Proof of Theorem 4 Let $u(t), v(t)$ be the solutions of (1.4), by (1.5), we have

$$
\lim _{t \rightarrow 1^{-}} \frac{u(t)}{v(t)}=1
$$

also notice that $f(t) \in \mathbb{R}_{q}, q>1$. Then for any $\varepsilon>0$, there exists $\delta>0$ such that

$$
(1-\varepsilon) v(t) \leq u(t) \leq(1+\varepsilon) v(t) \quad \text { and } \quad f(v(t))(1+\varepsilon) \leq f((1+\varepsilon) v(t)), \quad \text { for } t \in(1-\delta, 1)
$$

set $\bar{u}(t)=(1+\varepsilon) v(t)$, then

$$
\bar{u}^{\prime}\left(\frac{1}{2}\right)=u^{\prime}\left(\frac{1}{2}\right)=0 \quad \text { and } \quad u(t) \leq \bar{u}(t), \quad \text { for } t \in(1-\delta, 1) .
$$

We claim that for any $t \in\left[\frac{1}{2}, 1\right), u(t) \leq \bar{u}(t)$. Notice that

$$
\bar{u}^{\prime \prime}(t)=(1+\varepsilon) v^{\prime \prime}(t)=(1+\varepsilon) f(v(t)) \leq f(\bar{u}(t))
$$

from Lemma 7, the above claim is obvious. Similarly, we can prove that for any $t \in\left[\frac{1}{2}, 1\right)$, $u(t) \geq \underline{u}(t)$, where $\underline{u}(t)=(1-\varepsilon) v(t)$. Passing to the limit $\varepsilon \rightarrow 0^{+}$, we conclude that $u(t) \equiv$ $v(t)$.

Remark From the proof of Theorem 3 and Theorem 4, we can easily see that the condition $\frac{f(u)}{u}$ is increasing on $(0,+\infty)$ and $f(t) \in \mathbb{R}_{q}, q>1$ can be replaced by the following.

(H) There exists $q>1$ such that for any $\varepsilon>0$ sufficiently small, the function $f$ satisfies

$$
f[(1+\varepsilon) s] \geq(1+\varepsilon)^{q} f(s) \quad \text { and } \quad f[(1-\varepsilon) s] \leq(1-\varepsilon)^{q} f(s), \quad s \in(0,+\infty)
$$




\section{Competing interests}

The authors declare that there is no conflict of interests regarding the publication of this paper.

\section{Authors' contributions}

All authors contributed equally to this work. All authors read and approved the final manuscript.

\section{Acknowledgements}

The authors thank the reviewers for their insightful and detailed comments. This work is supported by NSF of China

(11201213, 11371183), NSF of Shandong Province (ZR2015AM026, ZR2013AM004) and the Project of Shandong Provincial Higher Educational Science and Technology (J15LI07).

Received: 23 March 2016 Accepted: 22 August 2016 Published online: 01 September 2016

\section{References}

1. Bieberbach, L: $\Delta u=e^{u}$ und die automorphen Funktionen. Math. Ann. 77, 173-212 (1916)

2. Keller, JB: On solutions of $\Delta u=f(u)$. Commun. Pure Appl. Math. 10, 503-510 (1957)

3. Osserman, R: On the inequality $\Delta u>f(u)$. Pac. J. Math. 7, 1641-1647 (1957)

4. Cîrstea, FC, Rădulescu, V: Uniqueness of the blow-up boundary solution of logistic equations with absorption. C. R. Math. Acad. Sci. Paris 335, 447-452 (2002)

5. Li, HL, Pang, PYH, Wang, MX: Boundary blow-up solutions of $p$-Laplacian elliptic equations with lower order terms. Z. Angew. Math. Phys. 63, 295-311 (2012)

6. Alsaedi, R, Maagli, H, Zeddini, N: Existence and global behavior of positive solution for semilinear problems with boundary blow-up. J. Math. Anal. Appl. 425, 818-826 (2015)

7. Wang, W, Gong, HZ, Zheng, SN: Asymptotic estimates of boundary blow-up solutions to the infinity Laplace equations. J. Differ. Equ. 256, 3721-3742 (2014)

8. Chen, YJ, Wang, MX: Boundary blow-up solutions of $p$-Laplacian elliptic equations with a weakly superlinear nonlinearity. Nonlinear Anal., Real World Appl. 14, 1527-1535 (2013)

9. García-Melián, J, Sabina de Lis, JC: A boundary blow-up problem with a nonlocal reaction. Nonlinear Anal. 75 2774-2792 (2012)

10. Mohammed, A, Mohammed, S: Boundary blow-up solutions to degenerate elliptic equations with non-monotone inhomogeneous terms. Nonlinear Anal. 75, 3249-3261 (2012)

11. Anuradha, V, Brown, C, Shivaji, R: Explosive nonnegative solutions to two point boundary value problems. Nonlinear Anal. 26(3), 613-630 (1996)

12. Wang, $\mathrm{SH}$ : Existence and multiplicity of boundary blow-up nonnegative solutions to two-point boundary value problems. Nonlinear Anal. 42(2), 139-162 (2000)

13. Zhang, ZJ: Existence of explosive solutions to two-point boundary value problems. Appl. Math. Lett. 17, 1033-1037 (2004)

14. Seneta, E: Regularly Varying Functions. Lecture Notes in Math., vol. 508. Springer, Berlin (1976)

\section{Submit your manuscript to a SpringerOpen ${ }^{\circ}$ journal and benefit from:}

- Convenient online submission

Rigorous peer review

- Immediate publication on acceptance

Open access: articles freely available online

- High visibility within the field

- Retaining the copyright to your article 